\title{
Impact of literature reports on drug safety signals
}

\author{
Bartlomiej Ochyra · Maciej Szewczyk · Adam Przybylkowski (iD
}

Received: 26 February 2020 / Accepted: 4 May 2020 / Published online: 26 May 2020

(C) The Author(s) 2020

\begin{abstract}
Summary
Background Signal management is considered an important activity in pharmacovigilance and should be performed using any available source of data, including scientific literature. The main aim of this study was to assess the role of scientific literature in both indexed and unindexed journals and compare the relevance of both in the signal management process.

Methods The study was a retrospective analysis of safety data. For the purposes of the study, drugs for which safety signals were evaluated by European Medicine Agency (EMA) were chosen. A match analysis of data collected in the EudraVigilance (EV) database with data from bibliographic databases such as MEDLINE, Embase or EBSCO (International Pharmaceutical Abstracts, IPA and the Allied and the Complementary Medicine Database, AMED) was performed.

Results A total of 73 drug event associations (DEA) and 4160 individual case safety reports (ICSRs) were analyzed. About 33\% of ICSRs were created based on scientific literature. A total of 1196 ICSRs were submitted to the EV database based on journals indexed in global bibliographic databases Embase $(86.00 \%)$ or MEDLINE (81.96\%) or EBSCO (IPA or AMED, $0.66 \%$ ). Conclusion This study underlines the importance of scientific literature for the signal management process in addition to other data sources. Most literature ICSRs from this analysis were created based on scientific journals indexed in bibliographic databases;
\end{abstract}

\author{
B. Ochyra $\cdot$ A. Przybylkowski $(\bowtie)$ \\ Department of Gastroenterology and Internal Diseases, \\ Medical University of Warsaw, Banacha 1a, 02-097 Warsaw, \\ Poland \\ aprzybylkowski@wum.edu.pl \\ M. Szewczyk \\ MS Pharm s.r.o., Praga, Czech Republic
}

therefore, it can be concluded that a systematic review of bibliographic databases, such as Embase or MEDLINE is highly relevant for the signal management process.

Keywords Drug safety evaluation - Literature search · Risk-benefit analysis - Bibliographic databases · Signal management

\section{Introduction}

Signal management is considered as an important activity in pharmacovigilance [1, 2]. Referring to Regulation (EU) No 520/2012 of 19 June 2012 the signal management process shall include the following activities: signal detection, signal validation, signal confirmation, signal analysis and prioritization, signal assessment, and recommendations for action [3].

Drug safety is assessed during both premarketing and postmarketing phases. Signal detection is a key step of the process, and a fundamental part of the postmarketing drug surveillance system [4]. The primary goal of this system is the timely detection of new and/or changed risks associated with an active substance or a medicinal product, for example a new adverse drug reaction (ADR) [5, 6]. A new aspect of a known risk (including known ADR) may change in severity, frequency, ADR outcome, duration, distribution (e.g. gender, age and country) and clinical nature [1].

Signals can arise from a wide variety of data sources. This includes all scientific information concerning the use of medicinal products and the outcome of the use, i.e. quality, non-clinical and clinical data (including pharmacovigilance and pharmacoepidemiological data). Signal detection should be performed using any available source of data, including data from the scientific literature [1]. 
The relevance of literature review in signal detection has been confirmed in many studies. For example, Klose et al. presented a relationship between floppy iris syndrome and tamsulin. The authors informed that during the same time period 13 cases were published in the literature, but none were spontaneously reported [5]. Such differences can be related to difficulties in identification and correct classification of some ADRs by consumers/patients. Based on the above example, it can be concluded that some safety concerns have little chance of detection based on spontaneous reports; however, it seems that healthcare professionals are more interested in publishing such reactions than reporting them spontaneously to the health authorities (HA) or marketing authorization holder (MAH) [7].

The literature is the source of individual case safety reports (ICSRs) and articles containing relevant safety information for inclusion in periodic safety update reports (PSUR) and/or emerging safety issue processes $[8,9]$. Many journals are not indexed in the most popular bibliographic databases. Information published in these journals can be a source of significant information relevant for the assessment of the risk-benefit balance. Because of that according to the guideline on good pharmacovigilance practices (GVP): Module VI, the MAH is expected to perform a systematic review of widely used reference databases and monitor scientific publications in local journals in countries where the medicinal products have marketing authorization [8].

Based on best knowledge, there has been no previous research comparing the relevance of the systematic review of journals indexed in bibliographic databases and unindexed journals for the signal management process. The main aim of this study was to assess the role of scientific literature in both indexed and unindexed journals and compare the relevance of both in the signal management process.

\section{Method}

The study was a retrospective analysis of publicly available data. For the purposes of the study, drugs for which safety signals were evaluated by the European Medicine Agency (EMA) between January 2016 and September 2018 were chosen. Only signals that led to product information (summary of product characteristics, SmPC and/or patient information lLeaflet, PIL) updates were analyzed. The combination of adverse event and drug was classified as a drug event association (DEA) and used for further analysis.

The relevant Pharmacovigilance Risk Assessment Committee (PRAC) recommendations on safety signals published on the EMA website (www.ema.europa. eu) were used to obtain a list of relevant drug international nonproprietary names (INNs) and the Medical Dictionary for Regulatory Activities (MedDRA) terms for each DEA. Preferred terms (PTs) for all signals were matched based on MedDRA version 21.1 for the purpose of line listing reports downloading process (line listing report provides listing of ICSRs for a specific substance and specific MedDRA terms).

As the next step, line listings were downloaded from the EudraVigilance (EV) database via the www. adrreports.eu website. The line listings were the source of data used for the assessment of the literature ICSR created based on both indexed and unindexed journals. The reference period for line listing (start date and end date of the period for reports taken into consideration during the analysis) was defined based on the EV gateway receipt date. The start date was specified as the date of first report for DEA identified in EV. The end date of the reference period was specified as the last day of the relevant PRAC meeting. A duplicate check process was performed to exclude possible duplicates.

A total of four bibliographic databases were selected for verification of literature ICRS: Embase, MEDLINE, EBSCO. The EBSCO covers a wide variety of resources, nevertheless only International Pharmaceutical Abstracts (IPA) and the Allied and the Complementary Medicine Database (AMED) were analyzed to imitate the process of medical literature monitoring performed by the EMA.

For each literature ICRS obtained from a line listing, these databases were analyzed to assess whether the journal which published a specific literature ICRS is indexed in the relevant database. During the next step, the collected data were analyzed using descriptive analysis.

\section{Results}

\section{Basic characteristics of analyzed DEAs}

In the period studied 82 DEAs were identified. For nine DEAs, there was no possibility to download relevant data from EV due to lack of data or problems with matching the proper PTs and/or INNs relevant for download of line listing. Therefore 73 DEAs were analyzed. The analyzed DEAs belonged to 12 different groups according to the anatomical therapeutic chemical (ATC) classification system.

Table 1 Source of individual case safety reports

\begin{tabular}{l|l|}
\hline Data source & Number of ICSRs \\
\hline Scientific literature (excluding scientific meetings) & 1206 \\
\hline Scientific meetings & 157 \\
\hline $\begin{array}{l}\text { Other spontaneous (excluding scientific literature and } \\
\text { scientific meetings) }\end{array}$ & 2796 \\
\hline \begin{tabular}{l} 
ICSR individual case safety report \\
\hline
\end{tabular}
\end{tabular}


Fig. 1 Time gap between first literature individual case safety report (ICSR) and spontaneous ICSR report. DEA drug event association

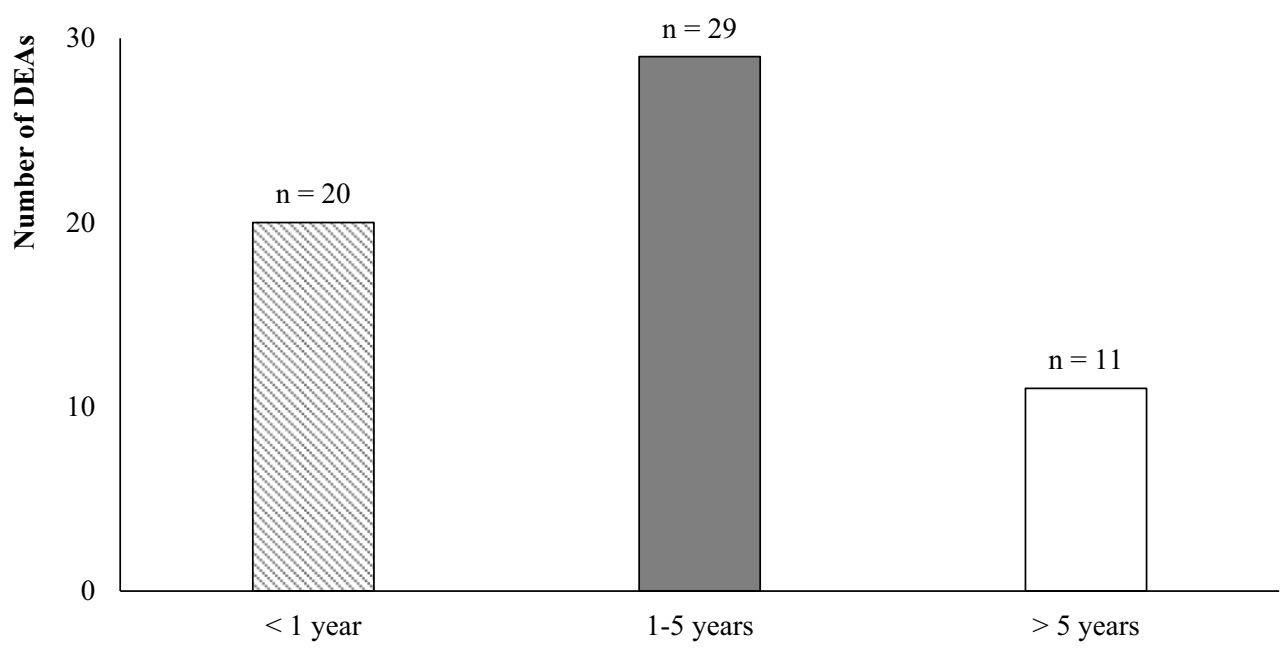

Time gap between first literature ICSR and spontaneous ICSR

Table 2 Indexation databases of literature individual case safety reports (ICSRs)

\begin{tabular}{|l|l|}
\hline Database & Number of ICSRs \\
\hline Embase & 1173 \\
\hline MEDLINE & 1118 \\
\hline EBSCO (IPA and AMED databases) & 9 \\
\hline Only in Embase & 79 \\
\hline Only in MEDLINE & 24 \\
\hline Only in EBSCO (IPA and AMED databases) & 9 \\
\hline $\begin{array}{l}\text { DEA drug event association, IPA international pharmaceutical abstracts, } \\
\text { AMED the allied and the complementary medicine database }\end{array}$
\end{tabular}

\section{Impact of data from scientific literature on signal management}

For the 73 analyzed DEAs, there were 4160 ICSRs retrieved from the EV. About 33\% of ICSRs originated from scientific literature (Table 1). For 13 DEAs there was no literature ICSRs. For 2 DEAs literature ICSRs constituted $100 \%$ of ICSRs submitted to the EV database during the analyzed period of time.
The highest number of literature ICSRs (499; $62.45 \%$ ) were reported for anti-infective drugs, while the lowest number of literature ICSRs (1;20\%) was noted for musculoskeletal system drugs.

The longest period between EV gateway receipt date (date of case receipt in EV) of first literature ICSR describing an analyzed adverse event and date of the first discussion at a PRAC meeting was 13 years. The shortest period between EV gateway receipt date of first literature ICSR describing an analyzed adverse event and signal discussion was 53 days.

For 12 DEAs the first ICSR was a literature ICSR. For 11 DEAs the first literature ICSR was indexed in Embase and MEDLINE, for 1 DEA the first literature ICSR was indexed only in Embase. Only for 1 DEA was the first literature ICSR not indexed in the analyzed bibliographic databases. The average delay between first ICSR from a source other than the literature and first literature ICSR was 38 months (Fig. 1). For 61 DEAs the first ICSR was a spontaneous report (other than the literature).
Fig. 2 Database of first literature individual case safety report (ICSRs) for drug event association (DEA). IPA international pharmaceutical abstracts, $A M E D$ the allied and the complementary medicine database

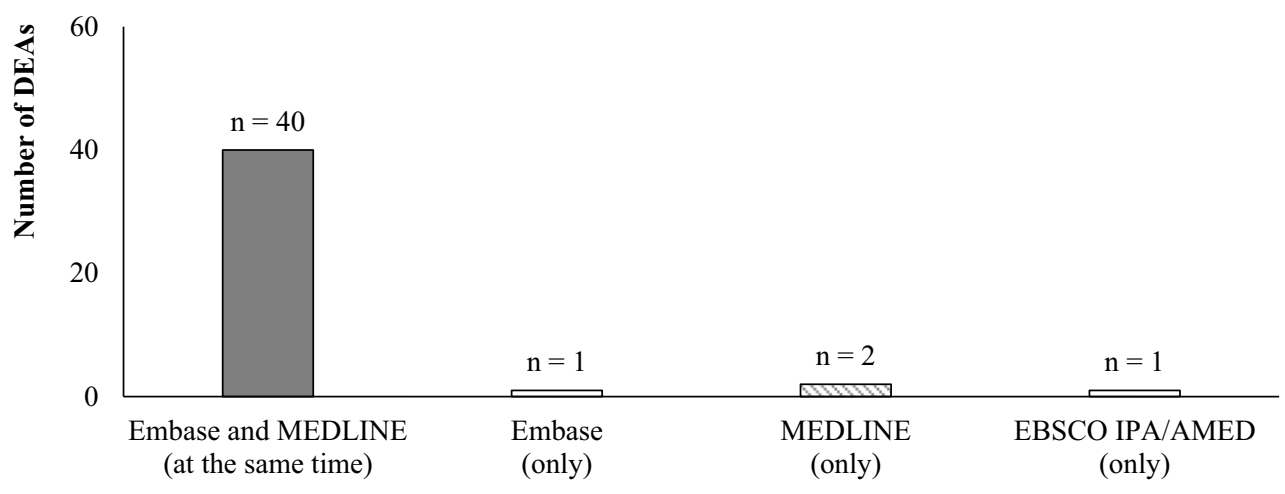

Name of database where first literature ICSR for DEA were published 
Fig. 3 Scientific meetcountry of literature individual case safety report (ICSRs) not indexed in Embase, MEDLINE, EBSCO (IPA/AMED) ing location or publication

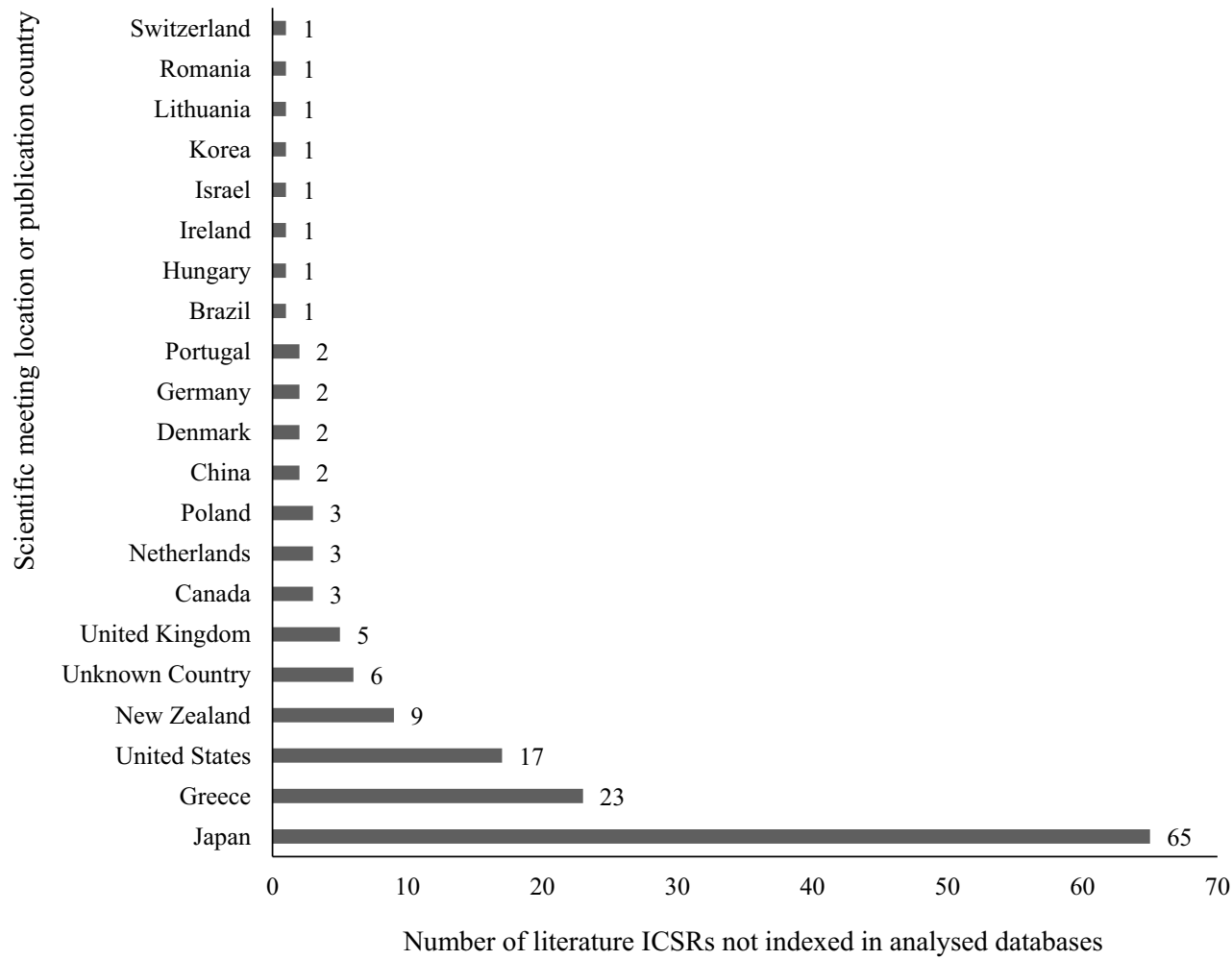

Impact of the analyzed bibliographic database on signal management

was a literature ICSR not indexed in any database the first report of an adverse event. This analysis confirms the importance of scientific literature as a source of data, in addition to other sources used for the signal management process, which is consistent with other authors' points of view [5, 9-12].

Klose et al. compared spontaneous and literature ICSRs. They assessed differences in the distribution of ADRs, quality of data, reporting rates of unexpected ADRs from both sources based on data from the German Health Authority (spontaneous ICSRs) and Vigilit database (literature ICSRs). Their study demonstrated the importance of literature ICSRs in addition to other spontaneous ICSRs for a sufficient risk-benefit assessment of medicinal products, and confirmed that none of these sources could be used as a stand-alone system in signal detection [5]. Pontes et al. made similar findings. Despite showing a few examples of literature relevance, such as the case of thalidomide-induced phocomelia, or nifedipine and fatal aplastic anemia, they assessed that the combination of spontaneous and literature ICSRs is necessary for the proper evaluation of drug safety because in some cases the drug's signal can be detected earlier through regular literature screening and other unexpected adverse events via spontaneous reporting systems [9].

In some cases, it may be reasonable to search information in journals that has a less clinical focus and includes more laboratory-based publications, especially during risk-benefit assessment of medicines that contain a new active substance [8]. The relevance of this type of information was confirmed by the signal 
for granulocyte macrophage colony-stimulating factor (GM-CSF) and increased risk of viral replication in acquired immune deficiency syndrome (AIDS) patients presented by Pontes et al. [9]. The first concerns about this risk were raised based on in vitro data [13, 14]. This type of safety signal would probably never have been detected based on spontaneous reports as the information came from in vitro studies published in scientific literature.

Based on our research, the systematic review of bibliographic databases, such as Embase or MEDLINE were assessed as the key during the literature screening process. Moreover, to ensure an effective signal management process, attention should be paid to term or text selection, to the approach to records retrieval and the application of limits. Garcelon et al. stated that the literature, specifically MEDLINE, is among the main sources of information used for signal detection [12]. Winnenburg et al. meanwhile confirmed that safety information extracted from MEDLINE is complementary to and not a replacement for other sources of information relevant for a risk-benefit assessment, but for effective literature screening knowledge about database structure is essential to prepare the proper search strategy and accurate retrieval of ADRs [7]. It is worth adding that signal detection based on scientific literature does not cause a delay in detection of a signal, which was confirmed by Shetty et al. They applied information mining to PubMed and discovered that 54\% of all detected FDA warnings used literature published before the warnings [15]. This is not consistent with this analysis, where the average delay between first ICSR from a source other than the literature and first literature ICSR was 38 months. This delay may be caused by the long journal review process. This can be also relevant when an author wants to publish a collection of cases. In such situations there could be a long gap between the first and last report. A variety of scientific databases are available and present several differences. Pontes et al. described and compared the databases and search engines relevant for periodic literature screening such as MEDLINE, Embase, Cochrane Library, CINAHL and PubMed, Ovid, ISI Web of Knowledge, ISI Web of Science, Scopus and Google Scholar. They showed the advantages and disadvantages of the most widely used databases. Nevertheless, due to differences such as coverage and accessibility, none of the available databases offers complete sensitivity for the literature screening. Based on this analysis, it is concluded that periodic literature searches should be performed in various carefully selected databases. Decisions about database selection, term or text selection, approach to records retrieval and the application of limits are highly relevant for an effective signal management process. Moreover, an MAH who knows the profile of their medicines can establish the most relevant source of published literature for each product, including the selection of the appropriate scientific database [8].

Based on best knowledge, there has been no previous research comparing the relevance of systematic reviews of journals indexed in bibliographic databases and unindexed journals for the signal management process. It was expected that the impact of unindexed literature (local journals) would be higher than was shown during the analysis of the data, due to its broader local scope and presence, broader data set (analysis of full-text articles instead abstracts) and the several times higher number of locally published journals than of journals indexed in databases. The hypothesis should be put forward that such a low rate of ICSRs from unindexed literature comes from the simple fact that such literature is not adequately screened, and thus not presented properly in the analyzed data. There are almost no required or recommended journal lists available across EU countries for local literature screening, thus each MAH has to assess and justify its own selection of journals without any standard limitation.

It should be also highlighted that 84 of 157 (53.50\%) ICSRs created based on information from various types of scientific meeting were not indexed in the analyzed reference databases, which is consistent with the information from appendix 2 of GVP module VI (revision 2) where it is stated that this type of information is a relevant source of data, but is often not available via scientific databases [8].

Moreover, it was noted that publications outside the EEA have a significant impact on the signal management process. For example, 65 literature ICSRs from Japan were not indexed in the analyzed scientific databases.

This study suffers from some limitations. Only those signals which were assessed by the PRAC were analyzed. Only ICSRs from EV were assessed, therefore ICSRs which were collected by an MAH and not sent to EV were not checked. Moreover, the quality of data provided by the literature ICSRs and their significance during the signal assessment step were not verified.

\section{Conclusion}

This study underlines the importance of the scientific literature for the signal management process, in addition to other data sources. Most literature ICSRs from this analysis were created based on scientific journals indexed in scientific databases, therefore it can be concluded that systematic review of scientific databases such as Embase or MEDLINE is highly relevant for the signal management process.

Funding Open Access funding provided by Medical University of Warsaw.

Conflict of interest B. Ochyra, M. Szewczyk, and A. Przybylkowski declare that they have no competing interests. 
Open Access This article is licensed under a Creative Commons Attribution 4.0 International License, which permits use, sharing, adaptation, distribution and reproduction in any medium or format, as long as you give appropriate credit to the original author(s) and the source, provide a link to the Creative Commons licence, and indicate if changes were made. The images or other third party material in this article are included in the article's Creative Commons licence, unless indicated otherwise in a credit line to the material. If material is not included in the article's Creative Commons licence and your intended use is not permitted by statutory regulation or exceeds the permitted use, you will need to obtain permission directly from the copyright holder. To view a copy of this licence, visit http://creativecommons.org/licenses/by/4.0/.

\section{References}

1. European Medicines Agency, Heads of Medicines Agencies. Guideline on good pharmacovigilance practices (GVP): moduleIX_signal management (rev 1). EMA/827661/2011 Rev1*.2017.

2. Pacurariu A, van Haren A, Berggren AL, Grundmark B, Zondag D, et al. SCOPE Work Package 5 signal management best practice guide. 2016.

3. European Commission. Commission implementing regulation (EU) no 520/2012 (4) on the performance of Pharmacovigilance activities provided for in regulation (EC) no 726/2004 and directive 2001/83/EC. Brussels: European Commission; 2012.

4. Ferreira GL, Shakir SS, Brown D. A systematic literature review of the determinants of signal detection in postmarketing surveillance systems. Drug Saf. 2008; https:// doi.org/10.2165/00002018-200831100-00038.

5. Klose J, Frohling S, Kroth E, Dobmeyer T, Nolting A. Safety information from spontaneous and literature adverse reaction reports: a comparison. Ther Innov Regul Sci. 2013;47(2):248-55. https://doi.org/10.1177/ 0092861512463920.

6. Egberts TC. Signal detection: historical background. Drug Saf. 2007;30(7):607-9. https://doi.org/10.2165/00002018200730070-00006.

7. Winnenburg R, Sorbello A, Ripple A, Harpaz R, Tonning J, Szarfman A, et al. Leveraging MEDLINE indexing for
pharmacovigilance-Inherent limitations and mitigation strategies. J Biomed Inform. 2015;57:425-35. https://doi. org/10.1016/j.jbi.2015.08.022.

8. European Medicines Agency, Heads of Medicines Agencies. Guideline on good pharmacovigilance practices (GVP): Module VI-Collection, management and submission of reports of suspected adverse reactions to medicinal products (Rev2). EMA/873138/2011 Rev2*. 2017.

9. Pontes H, Clement M, Rollason V. Safety signal detection: the relevance of literature review. Drug Saf. 2014;37(7):471-9. https://doi.org/10.1007/s40264-0140180-9.

10. Avillach P, Dufour JC, Diallo G, Salvo F, Joubert M, Thiessard F, et al. Design and validation of an automated method to detect known adverse drug reactions in MEDLINE: a contribution from the EU-ADR project. J Am Med Inform Assoc. 2013;20(3):446-52.https://doi.org/10.1136/ amiajnl-2012-001083.

11. Stricker BH, Psaty BM. Detection, verification, and quantification of adverse drugreactions. BMJ.2004;329(7456):44-7. https://doi.org/10.1136/bmj.329.7456.44.

12. Garcelon N, Mougin F, Bousquet C, Burgun A. Evidence in pharmacovigilance: extracting adverse drug reactions articles from MEDLINE to link them to case databases. Stud Health Technol Inform. 2006;124:528-33.

13. Folks TM, Justement J, Kinter A, Dinarello CA, Fauci AS. Cytokine-induced expression of HIV-1 in a chronically infected promonocyte cell line. Science. 1987;238(4828): 800-2.

14. Kitano K, Abboud CN, Ryan DH, Quan SG, Baldwin GC, Golde DW. Macrophage-active colony-stimulating factors enhance human immunodeficiency virus type 1 infection in bone marrow stem cells. Blood. 1991;77(8):1699-705.

15. Shetty KD, Dalal SR. Using information mining of the medical literature to improve drug safety. J Am Med Inform Assoc. 2011;18(5):668-74. https://doi.org/10.1136/ amiajnl-2011-000096.

Publisher's Note Springer Nature remains neutral with regard to jurisdictional claims in published maps and institutional affiliations. 